\title{
ALLOCATING VISUAL ATTENTION: HOW RELEVANCE TO DRIVING IMPACTS ATTENTION WHEN DRIVERS ARE DISTRACTED
}

\author{
Teena Garrison \\ Center for Advanced Vehicular Systems, Mississippi State University \\ Mississippi State, Mississippi, USA \\ Email: teenag@cavs.msstate.edu
}

\begin{abstract}
Summary: Use of cell phones while driving, and safety implications thereof, has captured public and scientific interest. Previous research has shown that driver reactions and visual attention are impacted by cell phone use, even when a handsfree device is used. In addition to these effects, Strayer and colleagues also found lower recognition for items present in the driving environment when drivers were using a cellular phone than when not using the phone. Strayer and colleagues used recognition as their primary measure of attention. Relevance to driving may have an impact on how attention is allocated to the environment via eye movements, separate from the impact on recognition memory. The current project used a medium-fidelity driving simulator to extend previous research by investigating how attention is allocated across driving-relevant and -irrelevant objects in the environment. Driving-relevant objects consisted of signs and potential hazards. Driving-irrelevant objects were billboards. Eye movement patterns (primarily measured by number of gazes) were impacted by distraction, and the pattern of gazes also differed across relevance levels, with hazards receiving the most gazes, and signs receiving the fewest. When only considering driving-relevant objects (i.e., signs vs. hazards), the eye movement measures showed an interaction between distraction and relevance. Signs received fewer gazes when drivers were distracted, whereas there was no comparable decrease in gazes to hazards.
\end{abstract}

\section{INTRODUCTION}

Cell phone use while driving has become a major concern of researchers, drivers and lawmakers. Numerous research studies have shown that driver reactions and attention are impacted by cell phone use, and legislative action has been taken in some states to limit drivers' use of cell phones (Governors Highway Safety Association, 2009). Additionally, systems are being developed by vehicle manufacturers to allow one's cell phone or 'smartphone' to be integrated with the vehicle controls. Cell phone use is becoming more truly 'hands-free' as voice dialing and speech recognition systems are both increasingly available and user-friendly. Since hand-held versus hands-free cell phone use is a major controversy in the driver distraction domain (e.g., Klauer, Dingus, Neale, Sudweeks, \& Ramsey, 2006; Strayer, Drews, \& Johnston, 2003), it is important to improve our understanding of how the cognitive distraction of a cell phone conversation may impact driver performance, separate from the manual and visual distractions involved in handheld phone use. Moving beyond cell phone use, an in-depth evaluation of the relationship between visual attention and driver performance may also allow more effective interface designs in situations that require driver multitasking, including cell phone-like conversations (e.g., emergency response and dispatch, military communications). 
Evaluating driver performance is often evaluating multitasking performance, because driving is a complex task. Many evaluations of driver performance involve more than what can be defined strictly as 'driving', that is, steering the vehicle in an intended direction and applying force to the accelerator and brake pedals until an intended location is reached. A driver may be following a colleague's car to an unfamiliar location, scanning an urban area for potential hazards, or discussing options for dinner in a cell phone conversation. Each situation requires shifting attention away from the 'primary' task of controlling the vehicle as the 'secondary' task requires attention and working memory resources. Because of its complexity, driving is often quite sensitive to distraction. A driver who attempts to monitor an intersection during a conversation with a passenger may overlook more visual information than a driver who is solely focused on his driving (e.g., McEvoy, Stevenson, \& Woodward, 2007). Additionally, some research indicates that a cell phone conversation may be even more disruptive to a driver's performance than is a conversation with a passenger (Drews, Pasupathi, \& Strayer, 2008).

Because most drivers are aware of the potential for distraction, one would anticipate that they aim to selectively use their cell phones when they perceive relatively undemanding driving conditions and avoid using their phones when demands are higher. Drivers may also attempt to compensate in a number of other ways for their distraction. They may attempt to restrict their attention to more driving-relevant information (e.g., other vehicles, interchanges) at the expense of less relevant information (e.g., roadway advertisements, buildings along the roadway; Richard, Wright, Ee, Prime, Shimizu, \& Vavrik, 2002) in addition to increasing the distance between their vehicle and others (e.g., Cooper \& Strayer, 2008). The current project was designed to investigate the potential impact of driving relevance on the allocation of visual attention when drivers were distracted by an ongoing conversation.

\section{METHOD}

\section{Participants}

Twenty licensed drivers from the Mississippi State University and local area population were paid \$20 for their participation (\$10 per hour for a maximum of 2 hours). This number was based on the Strayer et al. (2003) Experiment 3 and the accompanying effect sizes.

\section{Apparatus and Materials}

The driving simulator at the Center for Advanced Vehicular Systems includes a Nissan Maxima cab mounted on a six degree-of-freedom hexapod motion base. Three large screens provide approximately 180 degrees of visual angle to the front of the vehicle, and two built-in LCDs (side mirrors) and another screen placed behind the simulator provide an immersive virtual environment for driving scenarios. Due to limitations in the integration of the eye tracker with the simulator, the motion base functionality was not used for the current project. Communication between the experimenters and the participant occurred via an intercom system; the same system was used to simulate the hands-free cell phone in the distraction condition.

For the current project, the scenarios were stretches of four-lane highway, divided into three straight sections of approximately $2000 \mathrm{~m}$ each and connected by high-speed curves. The overall 
drive length was approximately 7000m and took roughly six minutes to complete. In addition to the static signs, hazards, and billboards used as stimuli, there was also light ambient traffic traveling the road with the simulated vehicle. The scenarios themselves were suburban areas transitioning from primarily residential to primarily commercial buildings or vice-versa.

Eye movements were recorded using a video-based, dash-mounted eye tracking system (faceLAB 4.6; Seeing Machines, Canberra, Australia). An infrared (IR) light source is mounted between the two cameras, allowing for precision tracking of the eye via the relationship between the pupil and the reflection of the IR light on the cornea. The faceLAB system has a sampling rate of $60 \mathrm{~Hz}$, and precision within approximately 0.5 degrees $\left(^{\circ}\right)$ of visual angle $\left(\sim 1^{\circ}\right.$ at the periphery). In its current configuration, the faceLAB system can accommodate approximately $30^{\circ}$ of viewing angle, allowing the participant some freedom of movement in the scene. Additionally, the faceLAB system can make less precise estimates outside the viewing angle (e.g., glances to a side mirror), based on head movement and tracking facial features.

\section{Experiment Design and Procedure}

Each participant completed two experiment drives, each containing three scenarios, which are defined as a straight section of road; high-speed curves separated the scenarios without disrupting the participant's experience of a single drive. Participants drove while having a casual conversation with an experimenter over a speaker/intercom system (to simulate hands-free driving, as with an integrated Bluetooth ${ }^{\circledR}$ system) for one blocked drive of scenarios and drove without a distracting task for the second blocked drive; the order of the drives were counterbalanced across participants.

As participants completed each experiment drive, they passed multiple critical objects at various points in each scenario. Critical objects were of three types: billboards, signs, or hazards. Replicating Strayer et al. (2003), billboards were used as ‘driving-irrelevant' objects. Additional environment objects were classified as signs or hazards. Road signs were used as drivingrelevant objects. Hazards included stalled and/or parked vehicles on the road shoulder and vehicles preparing to enter the roadway from side roads or driveways. Each scenario included two billboards (driving-irrelevant objects), two road signs (driving-relevant objects) and two potential hazards/events (hazard objects), resulting in six hazards, six signs, and six billboards per drive. Additional objects (primarily buildings) and light traffic were also added to make the environments feel more realistic and less sparse.

During the 'Distraction' drive, conversations were initiated before the beginning of the first scenario and continued throughout the drive; there was no manipulation of the simulated handsfree 'phone' necessary at any point during the drive. The 'Driving Only' drive provided a baseline for comparisons, to better determine the impact of distraction. Eye tracking measures included number of gazes and mean gaze duration. The faceLAB system (eye tracker) and driving simulator data sets were synchronized with video recorded by SimObserver. The integrated video and data files were then processed using Data Distillery 1.3, which supports frame-by-frame analysis and annotation of synchronized data files and video.

In addition to the eye movement measures, driving performance measures (i.e., lane position, steering angle, and velocity) were also collected. Speed and steering angle were found to be 
more variable when the driver was distracted, replicating results found in previous research (e.g., Kubose, Bock, Dell, Garnsey, Kramer, \& Mayhugh, 2006; Liang \& Lee, 2010).

A recognition test was also completed by participants, but only stimuli presented during the driving-only condition were remembered at a greater than chance level (0.59), compared to the distraction condition (0.49). These results replicate previous research by Strayer and colleagues (e.g., Strayer, et al., 2004); thus, the remainder of this paper will focus solely on the eye movement results.

\section{RESULTS}

Of the twenty participants who completed the current study, experimenter error resulted in one participant only having overall driver performance data. An additional participant was lost from the eye movement analyses due to eye tracking equipment error. The following analyses thus include data from eighteen participants. Measures of effect size are provided through the use of eta-squared $\left(\eta^{2}\right)$ for analyses of variance. A larger $\eta^{2}$ value indicates a stronger association between the independent variable and the dependent measure being considered.

\section{Demographics of Participants}

Twenty (12 male, 8 female) licensed drivers participated in the current project; participants averaged 24.4 years of age $(S D=, 6.3$ ) with a range of 18 to 42 years of age. Participants had been driving for an average of 6.8 years $(S D=7.0)$, and had on average 16.6 years of education $(S D=2.3)$. All participants owned a cellular phone, and $75 \%$ of them indicated that they used their phone while driving. Eighty percent of the participants indicated that they played video games, a common form of virtual reality or simulation technology.

\section{Impact of Relevance to Driving on Visual Attention}

Previous research by Strayer et al. (2003, see also Strayer, Cooper, \& Drews, 2004) found no difference in eye tracking patterns due to distraction; based on these results, it was expected that eye movement patterns would not differ due to distraction. However, it was expected that participants would look at hazards more frequently than at billboards, perhaps due to their task relevance and central proximity in the driving environment. Signs were expected to be intermediate to hazards and billboards in the number and duration of gazes. Figure 1 shows what proportion of each relevance category received gazes by distraction condition.

An ANOVA was conducted on mean proportion of critical objects receiving gazes with drive as a between-subject variable, and distraction and relevance as within-subject variables. In contrast with the Strayer et al. (2003) findings, and with my expectations, eye movement patterns differed between the driving-only and distraction conditions. There were main effects of distraction, $F(1$, $16)=4.52, p<.05, \eta^{2}=0.22$, and relevance, $F(2,32)=72.20, p<.0001, \eta^{2}=0.82$. There were no significant interactions, including distraction $\times$ relevance, $F(2,32)=2.41, p=.11$. Thus, there were fewer gazes toward the critical objects in the distraction condition, and hazards received more gazes than billboards, which received more gazes than signs. The expectations 
regarding relevance were thus partially supported, although the expectation for signs being intermediate to hazards and billboards was clearly not met.

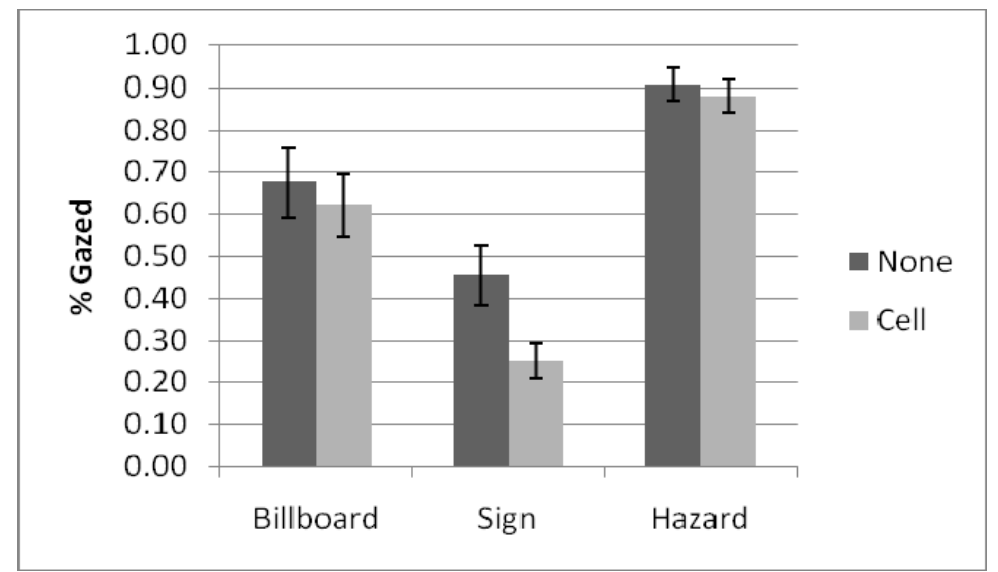

Figure 1. Proportion of critical objects receiving gazes for each relevance category by distraction condition (Error bars represent the standard error of the mean)

One potential explanation for why signs received fewer gazes than either billboards or hazards may be their smaller size in the environment. Signs may not attract attention as easily in the environment, they may be overlooked, or gazes that did occur on the signs may be harder to distinguish from nearby objects because of a combination in error in eye tracking recording and a relatively small target object. However, the limitations of the simulation system in presenting the signs compared to the larger billboards and hazards may also have played a role, because signs would 'pop in' closer to the participant's vehicle and be presented for a shorter period of time. This limitation must be addressed before specific conclusions regarding the signs can be drawn.

Although my original goal was to evaluate both mean number of gazes and mean gaze duration across the distraction and relevance manipulations, the resulting data contain too many missing values for duration to analyze successfully. No critical object received a gaze in all cases, and thus every critical object has some missing data for duration (i.e., there was no gaze to be counted for that object of any duration). Figure 2 shows the resulting mean durations for each distraction and relevance manipulation, labeled with the available number of observations. The maximum number of observations possible per cell is eighteen. The available data suggest that gaze durations were shorter for the distraction condition than for the driving-only condition.

An ANOVA was conducted on the gaze duration means that were available, resulting in 11 (of 18) participants being included, with drive as a between-subject variable, and distraction and relevance as within-subject variables. The only significant main effect was relevance, $F(2,18)=$ $9.65, p<.01, \eta^{2}=0.47$. In contrast to the results for proportion of gazes, the pattern of the gaze duration data follows the expected progression from billboards, to signs, to hazards in increasing duration. Thus, there is some evidence that driver eye movement patterns, and thus driver attention, are sensitive to objects' relevance to the driving task. It is possible that a distraction effect might be precluded by a lack of power due to the limited number of participants, because there is a clear trend toward shorter gazes in the cell phone distraction condition. 


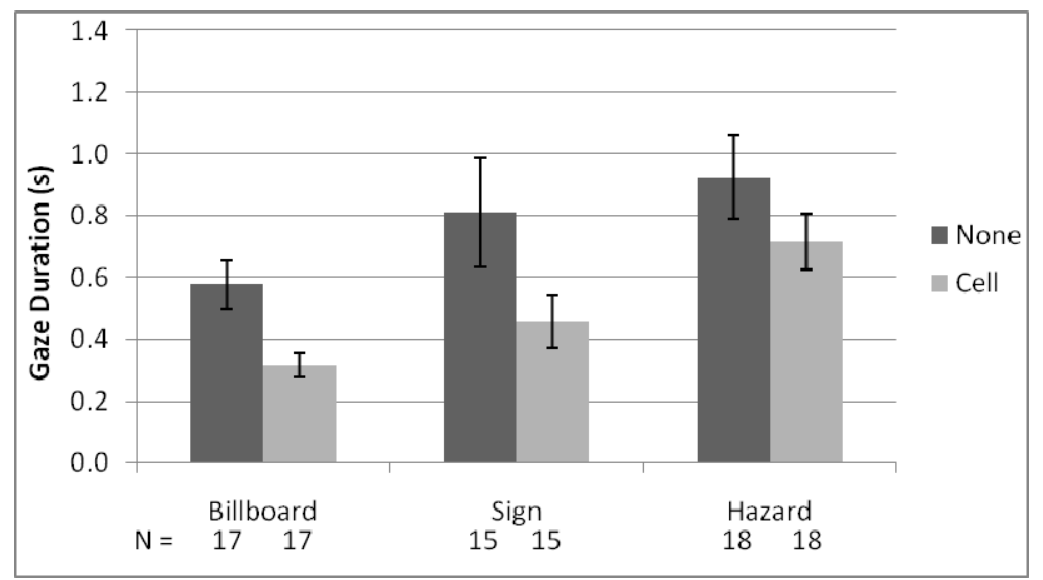

Figure 2. Mean gaze duration (in seconds) for critical objects by distraction condition and relevance for all participants (Error bars represent the standard error of the mean; N values indicate actual number of participants who gazed on at least one critical object for each relevance category)

\section{CONCLUSIONS}

The presented research was conducted in an effort to extend current understanding of how visual attention is impacted by distraction, and to clarify the potential impact of relevance to driving on attention allocation. In addition to the apparent differences in eye movement measures due to distraction, eye movement measures were also found to vary based on driving relevance.

Evidence that eye movement measures differ between objects that are relevant versus irrelevant to the driving task supports previous results (e.g., Henderson, Malcolm \& Schandl, 2009), indicating that attention is primarily allocated in a top-down manner and suggesting that the driving task maintained priority in the presence of another attention-demanding task. Further, there were also differences in how attention was allocated to hazardous events compared to other less relevant objects, suggesting that drivers attempted to compensate for the distraction by attending to the most driving-relevant and potentially most dangerous aspects of the environment. However, because the roadside hazards in the current project never materialized as actual hazards (i.e., the vehicles never entered the driving lane), driver responses to potential hazards, and what role attention allocation and distraction may play in these situations, cannot be evaluated.

\section{ACKNOWLEDGMENTS}

The presented research has previously been presented as part of the author's doctoral dissertation in Cognitive Science at Mississippi State University.

\section{REFERENCES}

CAVS Driving Simulator [Apparatus and software]. Royal Oak, MI: Realtime Technologies, Inc.

Cooper, J. M. \& Strayer, D. L. (2008). Effects of simulator practice and real-world experience on cell-phone-related driver distraction. Human Factors, 50, 893-902.

Data Distillery (Version 1.3) [Computer software]. Fort Collins, CO: Tumo Kinetic, LLC. 
Drews, F. A., Pasupathi, M., \& Strayer, D. L. (2008). Passenger and cell phone conversations in simulated driving. Journal of Experimental Psychology: Applied, 14, 392-400.

FaceLAB (Version 4.6) [Apparatus and software]. Canberra, Australia: Seeing Machines.

Governors Highway Safety Association. (2010, October). State cell phone driving laws. Retrieved October 7, 2010, from http://www.statehighwaysafety.org/html/stateinfo/laws/cellphone_laws.html.

Henderson, J. M., Malcolm, G. L., \& Schandl, C. (2009). Searching in the dark: Cognitive relevance drives attention in real-world scenes. Psychonomic Bulletin and Review, 16, 850856.

Klauer, S. G., Dingus, T. A., Neale, V. L., Sudweeks, J. D., \& Ramsey, D. J. (2006). The impact of driver inattention on near-crash/crash risk: An analysis using the 100-car naturalistic driving study data. Washington, DC: National Highway Traffic Safety Administration. (NTIS No. DOT HS 810 594)

Kubose, T. T., Bock, K., Dell, G. S., Garnsey, S. M., Kramer, A. F., \& Mayhugh, J. (2006). The effects of speech production and speech comprehension on simulated driving performance. Applied Cognitive Psychology, 20, 43-63.

Liang, Y. \& Lee, J. D. (2010). Combining visual and cognitive distraction: Less than the sum of its parts. Accident Analysis and Prevention, 42, 881-890.

McEvoy, S. P., Stevenson, M. R., \& Woodward, M. (2007). The contribution of passengers versus mobile phone use to motor vehicle crashes resulting in hospital attendance by the driver. Accident Analysis and Prevention, 39, 1170-1176.

Richard, C. M., Wright, R. D., Ee, C., Prime, S. L., Shimizu, Y., \& Vavrik, J. (2002). Effect of a concurrent auditory task on visual search performance in a driving-related image-flicker task. Human Factors, 44, 108-119.

SimObserver [Computer software]. Royal Oak, MI: Realtime Technologies, Inc.

Strayer, D. L., Cooper, J. M., \& Drews, F. A. (2004). What do drivers fail to see when conversing on a cell phone? Proceedings of the 48nd Annual Meeting of the HumanFactors and Ergonomics Society, 2213-2217.

Strayer, D. L., Drews, F. A., \& Johnston, W. A. (2003). Cell phone-induced failures of visual attention during simulated driving. Journal of Experimental Psychology: Applied, 9, 23-32. 